\section{Digital reader vs print media: the role of digital technology in reading accuracy in age-related macular degeneration}

K Gill, A Mao, AM Powell and T Sheidow

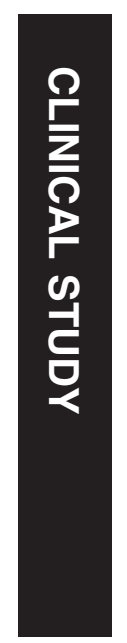

\begin{abstract}
Purpose To compare patient satisfaction, reading accuracy, and reading speed between digital e-readers (Sony eReader, Apple iPad) and standard paper/print media for patients with stable wet age-related macular degeneration (AMD).

Methods Patients recruited for the study were patients with stable wet AMD, in one or both eyes, who would benefit from a lowvision aid. The selected text sizes by patients reflected the spectrum of low vision in regard to their macular disease. Stability of macular

with the Sony eReader $(P<0.03)$ in all text group sizes. Patients chose the iPad to have the best clarity and the print paper as the easiest to use.

Conclusions This study has demonstrated that digital devices may have a use in visual rehabilitation for low-vision patients.

Devices that have larger display screens and offer high contrast ratios will benefit AMD patients who require larger texts to read. Eye (2013) 27, 639-643; doi:10.1038/eye.2013.14; published online 15 March 2013
\end{abstract} degeneration was assessed on a clinical examination with stable visual acuity.

Patients recruited for the study were assessed for reading speeds on both digital readers and standard paper text. Standardized and validated texts for reading speeds were used. Font sizes in the study reflected a spectrum from newsprint to large print books. Patients started with the smallest print size they could read on the standardized paper text. They then used digital readers to read the same size standardized text. Reading speed was calculated as words per minute by the formula (correctly read words/reading time (s) $\cdot 60$ ). The visual analog scale was completed by patients after reading each passage. These included their assessment on 'ease of use' and 'clarity of print' for each device and the print paper.

Results A total of 27 patients were used in the study. Patients consistently read faster $(P<0.0003)$ on the Apple iPad with larger text sizes (size 24 or greater) when compared with paper, and also on the paper compared
Keywords: low vision; macular degeneration; reading speed/accuracy; digital devices

\section{Introduction}

Age-related macular degeneration (AMD) is a disease of the central macular function that has significant and severe impact on a patient's central acuity, resulting in a dramatic decrease in the ability with which they accurately read and the speed with which reading occurs. ${ }^{1}$ In the past several years, the use of antivascular endothelial growth factor agents has greatly advanced our management of AMD and has had a profound impact on visual outcomes for patients with these diseases..$^{2,3}$ Studies such as $\mathrm{ANCHOR}^{4}$ and MARINA ${ }^{5}$ have shown significant benefits and improvement in patient's quality of life following treatment of wet AMD. Although improvement in quality of life can be achieved, these improvements are relative to the baseline level of visual acuity and unfortunately do not represent a return to
Department of

Ophthalmology, University of Western Ontario, London,

Ontario, Canada

Correspondence:

K Gill, Ivey Eye Institute, University of Western, St Joseph's Hospital, 268 Grosvenor Street, PO Box 5777, London, Ontario N6A 4V2, Canada Tel: +1 519859 9719; Fax: +1 5196466248 E-mail: kulbir.gill@ gmail.com

Received: 25 September 2012

Accepted in revised form: 24 January 2013

Published online: 15 March 2013

Previously presented at the Canadian Ophthalmology Society Conference 2012 in Toronto, Ontario, Canada 
normal function. As such, many patients treated for wet macular degeneration are left with vision that does not allow them to read in a normal manner.

The general public, non-ophthalmic physicians, and ophthalmologists who treat patients with AMD markedly underestimate the quality-of-life loss associated with this condition. ${ }^{6}$ Patients with moderate AMD reported a $40 \%$ decrease in quality of life, with very severe AMD patients reporting a decline of $63 \%$ in quality of life. ${ }^{6}$

The impact on quality of life has been found to be independently associated with vision loss. ${ }^{7}$ It is recommended that patients with mild AMD (visual acuity worse than 6/12) can have significant improvements in their quality of life with a referral to a low-vision service. ${ }^{7}$

Three studies related to the epidemiology of vision impairment list macular degeneration as the leading cause of vision impairment in the developed world. ${ }^{8-10}$ Most low-vision clinics attract AMD patients as they tend to notice a decline in central vision acuity more quickly and it has detrimental effects on everyday visual activities. $^{11}$

The Wilmer low-vision clinic study showed that $64 \%$ of patients indicated that 'reading' was their chief complaint, whereas other activities were identified in $<8 \%$ of patients. Thus, most AMD patients are referred to low-vision clinics with a concern of reading. ${ }^{11}$

Reading rehabilitation in low-vision clinics relies on tools that can measure reading speed effectively and have a high index of reliability. Both the Minnesota Reading Test (MNREAD) and Radner devices contain single and relatively short sentences to gauge reading acuity and magnification needs. However, both tests lack longer text passages to effectively measure and record improvements in reading speed. ${ }^{12}$ More recently, reading passages with standardized texts were created to assess reading speed during repeated measurements and across languages for normal subjects and low-vision patients. ${ }^{12}$ Through the Hahn group, standardized texts to measure reading speeds were developed and named International Reading Speed Text (iREST). These passages were developed in Germany in 2006 and have been standardized for content (reading material at a sixth grade level), length $(830 \pm 2$ characters $)$, syntactic complexity, and spacing. These reading texts have been proven to be an invaluable diagnostic tool in low-vision research and in the comparison of different low-vision reading aids in visual rehabilitation in patients with AMD. ${ }^{12,13}$

Low-vision studies demonstrate that patients receive benefits from magnification, improved contrast, and augmented lighting, all of which may impact the patient's ability to read. Recently, digital technology products have become available to access the growing online digital media world. Only about $1.5 \%$ of the approximately two million books currently in print are available in large print format. ${ }^{13}$

Books, magazines, and online articles are becoming more available for patients, increasing access to those who are able to utilize them. Furthermore, these digital readers have the ability to modify the degree of brightness and contrast, as well as the size of the type, making them ideal to consider for patients with some lower visual needs. Electronic books as visual aids have been postulated by many low-vision experts as the next wave in visual rehabilitation with our aging population. ${ }^{14,15}$

The purpose of this study was to compare patient satisfaction, reading accuracy, and reading speed between digital e-readers (Sony eReader (Sony, New York, NY, USA), Apple iPad (Apple, Cupertino, CA, USA)) and standard paper/print media.

\section{Methods}

Patients recruited for the study were patients with stable wet AMD, in one or both eyes, who would benefit from a low-vision aid based on ocular assessment by their retinal specialist. Stability of macular degeneration was assessed on a clinical examination with stable visual acuity.

Standardized texts validated through the Hahn study for reading speeds were used. Three different text contents were used and assigned to the print paper group, the Sony eReader group, and the Apple iPad group. The font chosen for all passages was New Courier, as mono-spaced and serif-type font were found to assist reading performance in patients with AMD. ${ }^{16}$

Text sizes chosen were size 12, 16, 24, 32, 50, and 80 for the standard print group. Selected text sizes by patients reflected spectrum of low vision in regards to their macular disease. The selected text sizes for each patient reflected their visual acuity status. These font sizes were equilibrated to both digital readers with the use of a microcaliper to ensure equal font sizes across all devices. These font sizes allowed for the spectrum of fine newsprint to large print books.

Patients recruited for the study were assessed for reading speeds on both digital readers and standard paper text. All reading assessments were carried out in the same room to account for room luminance.

Patients started with the smallest print size they could comfortably read on the standardized paper text. The paper text was used first to ensure patients could comfortably read their selected text size with ease and comfort before using the digital readers. The digital readers were then used to read the same size 
standardized text on the digital readers. The digital readers were calibrated to default contrast. Patients were given teaching on digital readers by a technician before utilizing the electronic readers in the study, including how to scroll through text during their reading sessions and how to adjust contrast and magnification outside the reading assessment.

The Apple iPad used was the original iPad with a 9.7-inch (diagonal) LED-backlit screen that is 1024-by768-pixel resolution at 132 pixels per inch (ppi). The Sony eReader used was the PRS-T1 model with a 6-inch diagonal screen with a 16-level gray scale display.

Each patient was asked to read the standardized randomized texts aloud on each of the digital readers and the paper text. The subjects were instructed to read aloud as fast as they could without making mistakes and without correcting mistakes. Oral reading times were measured. To evaluate reading performances, standardized text of equal length and comparable difficulty (on both standard paper text and digital readers) were used. The wrongly read words are noted and subtracted from the total number of words within the text. Reading speeds were calculated in words per minute (WPM) (correctly read words/reading time $(\mathrm{s}) \cdot 60) .{ }^{13}$

Demographic information such as age, gender, and past ocular history was also recorded.

Patients completed a visual analog scale after reading passages on each medium. These included their assessment on ease of use and clarity of print on each device and for the print paper.

\section{Results}

In total, we had 27 patients in our study. Their average age was 78.9 years old. Their visual acuity in the eye with stable wet AMD ranged from 20/25 to CF.

Table 1 shows the mean reading speeds published by iREST on normal individuals compared with that of patients in the study using the various devices. The average reading speed on paper was 113.2 WPM, on the Sony eReader was 110.6 WPM, and on the Apple iPad was 117.1 WPM. Note that there is a high standard deviation for our patient subset because of their varying visual acuities at near.

We then subdivided our patients into three groups based on the text size they chose to read with. These text-size-based groups helped to divide the patient population into their respective functional vision at near. Table $1 \mathrm{~b}$ shows the text sizes used to divide the patients.

We observed that the reading speeds declined $(P<0.0001)$ for those using larger fonts to read the standardized passages across all three devices.
Table 1 (a) Average reading speed for patients on each device compared with baseline reading speed for the normal population for standardized iREST text passages; (b) patient groupsbased text size they chose to read with; (c) mean change in reading speeds when comparing Apple iPad with paper print across all three text groups; (d) mean change in reading speeds when comparing Sony eReader with paper print across all three text groups

\begin{tabular}{lcc}
\hline (a) & & \\
Device & Average WPM & SD \\
\hline Paper & 113.2 & 50.1 \\
Apple iPad & 117.1 & 49 \\
Sony eReader & 110.6 & 49.1 \\
iREST baseline & 243 & 28
\end{tabular}

(b)

\begin{tabular}{lcc} 
Group & Text size & Number of patients \\
\hline 1 & $12-16$ & 9 \\
2 & 24 & 9 \\
3 & $\geq 32$ & 9
\end{tabular}

(c)

\begin{tabular}{lccc} 
Text size & $\begin{array}{c}\text { Number of } \\
\text { patients }\end{array}$ & $\begin{array}{c}\text { Mean change in WPM } \\
\text { (iPad-paper) }\end{array}$ & P-value \\
\hline $12-16$ & 9 & +0.62 & 0.52 \\
24 & 9 & +5.85 & 0.0003 \\
$\geq 32$ & 9 & +5.27 & $<.0001$
\end{tabular}

(d)

\begin{tabular}{lccc} 
Text size & $\begin{array}{c}\text { Number of } \\
\text { patients }\end{array}$ & $\begin{array}{c}\text { Mean change in WPM } \\
\text { (eReader-paper) }\end{array}$ & P-value \\
\hline $12-16$ & 9 & -2.62 & 0.02 \\
24 & 9 & -3.7 & 0.01 \\
$\geq 32$ & 9 & -1.34 & 0.03 \\
\hline
\end{tabular}

Regardless of what device was used-patients with low vision consistently read slower on all three devices (Figure 1).

For each patient, we utilized the reading speed with print paper as the baseline reading speed. Comparison of this baseline to reading speeds on the Apple iPad and Sony eReader were then made. This allowed for the comparison of changes in reading speeds for each individual patient across the three reading devices.

Table 1c shows the change in reading speeds comparing the Apple iPad with print paper across all three text groups. With larger print sizes (text size 24 or greater), patients' mean reading speeds were faster on the Apple iPad when compared with print paper.

Table 1d shows changes in reading speeds when comparing the Sony eReader with print paper across all 


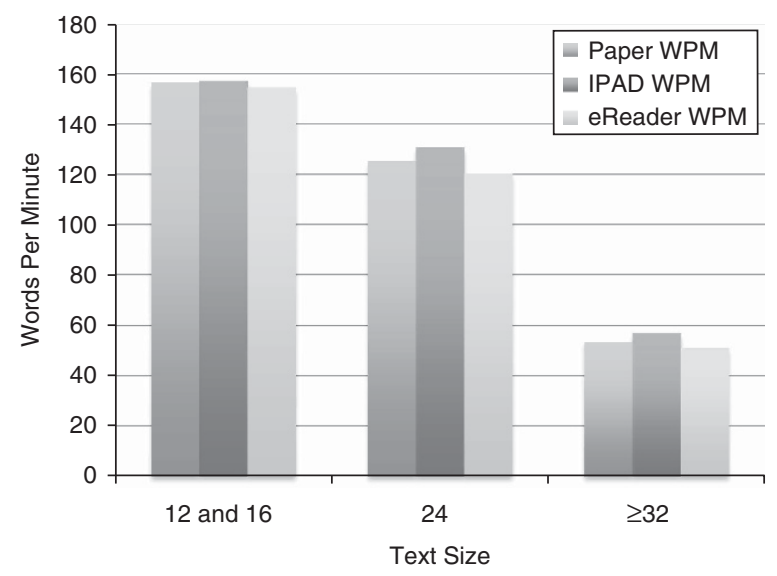

Figure 1 Reading speeds decreased when patients selected larger print size across all three devices $(P<0.0001)$.

three text groups. In all three text groups, patients' mean reading speeds were faster on paper print when compared with the Sony eReader.

For the visual analog scale (Figures 2 and 3), patients rated paper print as being the easiest device to use and rated the Apple iPad as having the best clarity.

\section{Discussion}

This novel study was designed to examine the use of digital readers as tools in visual rehabilitation. We were able to take standardized and validated texts for reading speeds and apply them to AMD patients.

The limitation in this study was the variation in near vision among our patient population set. Many patients had only AMD in one eye, but were tested binocularly. Furthermore, although validated and standardized texts were used for reading speeds, each patient had varying degrees of correction at near vision. To account for this, we simply divided patients into three subsets based on the text size they felt most comfortable reading. This allowed us to stratify our patients according to their measured low vision.

By making the patients' reading speed with standard paper print as their baseline, we were able to compare each patient's individual baseline reading speed with that on the Apple iPad and Sony eReader. We observed that patients consistently read faster $(P<0.0003)$ on the Apple iPad with larger text sizes (text size of 24 or greater) when compared with paper. These findings may be related to the higher contrast on the Apple iPad $(670: 1)$ and its bright background. This allows patients with decreased contrast sensitivity and central vision from AMD to read faster on the Apple iPad compared with paper.

Patients consistently read faster on the paper compared with the Sony eReader $(P<0.03)$ in all text

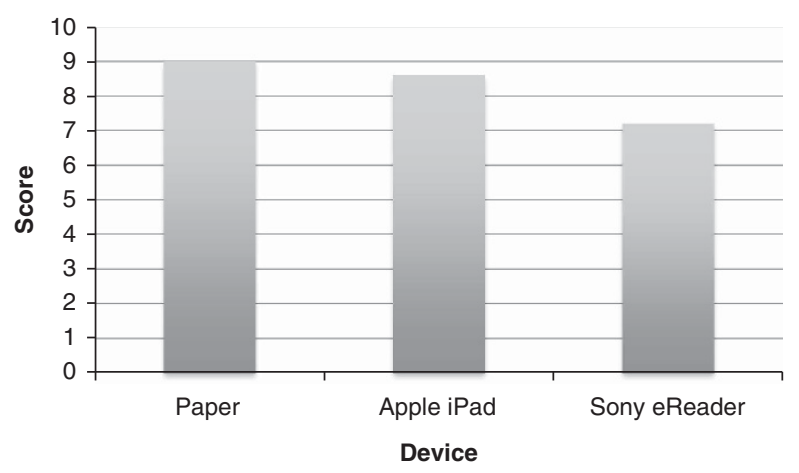

Figure 2 Patient survey on 'ease of use' for each device $(P<0.001)$.

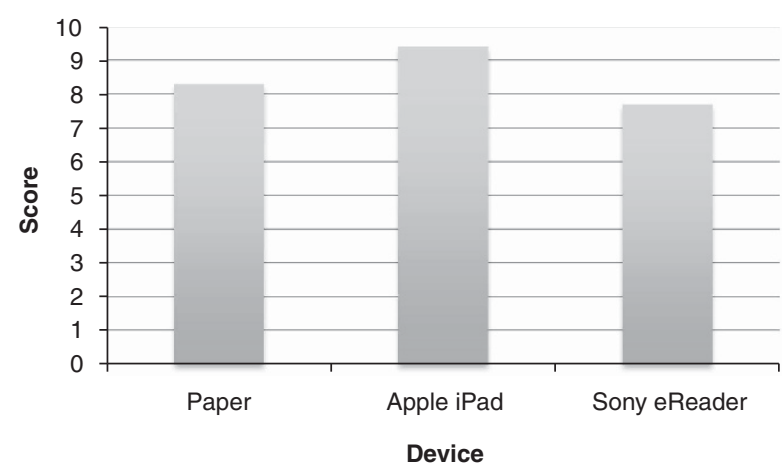

Figure 3 Patient survey on 'clarity of text' for each device $(P<0.001)$.

group sizes. The monochromatic gray scale of the eReader did not provide as much contrast when compared with the black on white displays of the Apple iPad and the standard paper. The screen of the eReader is much smaller (diagonal length of 6 in) compared with the standard paper on letter size and the Apple iPad (diagonal length of $9.7 \mathrm{in}$ ). This may account for why patients read slower on this device, as they constantly had to spend time scrolling through pages with larger text sizes.

Through this study, we observed that patients preferred the ease of use of paper when compared with the digital readers. This may be attributed to the patients' age and their lack of exposure to technology. However, patients did prefer the clarity of text on the Apple iPad compared with both paper and the Sony eReader. This can be attributed to its high contrast display setting.

This study has demonstrated that digital devices may have a use in visual rehabilitation for low-vision patients. Devices that have larger display screens and offer high contrast ratios will benefit AMD patients who require larger texts to read. 


\section{Summary}

\section{What was known before}

- Digital devices may provide better magnification for lowvision patients.

- Patients with low vision from AMD have difficulty with reading text.

\section{What this study adds}

- With a validated text (iREST) patients with low vision from AMD benefit from using digital devices for reading.

\section{Conflict of interest}

The authors declare no conflict of interest.

\section{References}

1 Khandhadia S, Cherry J, Lotery AJ. Age-related macular degeneration. Adv Exp Med Biol 2012; 724: 15-36.

2 Zechmeister-Koss I, Huic M. Vascular endothelial growth factor inhibitors (anti VEGF) in the management of diabetic macular oedema: a systematic review. Br J Ophthalmol 2012; 96: 167-178.

3 Keane PA, Sadda SR. Development of anti-VEGF therapies for intraocular use: a guide for clinicians. J Ophthalmol 2012; 483034.

4 Brown DM, Michels M, Kaiser PK, Heier JS, Sy JP, Ianchulev T. ANCHOR Study Group. Ranibizumab versus verteporfin photodynamic therapy for neovascular age-related macular degeneration: two year results of the ANCHOR study. Ophthalmology 2009; 116(1): 57-65.e5.5.

5 Bressler NM, Chang TS, Suner IJ, Fine JT, Dolan CM, Ward J et al. MARINA and ANCHOR Research Groups. Visionrelated function after ranibizumab treatment by better- or worse-seeing eye: clinical trial results from MARINA and ANCHOR. Ophthalmology 2010; 117(4): 747-756.e4.

6 Brown M, Brown G, Sharma S, Stein J. The burden of age-related macular degeneration: a value-based analysis. Curr Opin Ophtalmol 2006; 17: 257-266.

7 Hassell JB, Lamoureux EL, Keeffe JE. Impact of age related macular degeneration on quality of life. $\mathrm{Br} J$ Ophthalmol 2006; 90: 593-596.

8 Dana MR, Tielsch JM, Enger C, Joyce E, Santoli JM, Taylor HR. Visual impairment in a rural Appalachian community: prevalence and causes. JAMA 1990; 264: 2400-2405.

9 Rahmani B, Tielsch JM, Katz J, Gottsch J, Quigley H, Javitt J et al. The cause-specific prevalence of visual impairment in an urban population: the Baltimore Eye Survey. Ophthalmology 1996; 103: 1721-1726.

10 Attebo K, Mitchell P, Smith W. Visual acuity and the causes of visual loss in Australia: the Blue Mountains Eye Study. Ophthalmology 1996; 103: 357-364.

11 Rubin G. Vision rehabilitation for patients with age related macular degeneration. Eye 2001; 15: 430-435.

12 Hahn GA, Penka D, Gehrlich C, Messias A, Weismann M, Hyvärinen L et al. New standardised texts for assessing reading performance in four European languages. $\mathrm{Br} J$ Ophthalmol 2006; 90: 480-484.

13 Nguyen N, Weismann M, Trauzettel-Klosinski S. Improvement of reading speed after providing of low vision aids in patients with age-related macular degeneration. Acta Ophthalmol 2009; 87: 849-853.

14 Lockyer S, Creaser C, Davies JE. Availability of accessible publications: designing a methodology to provide reliable estimates for the right to read alliance. Health Info Libr J 2005; 22: 243-252.

15 Crossland MD, Macedo AF, Rubin GS. Electronic books as low vision aids. Br J Ophthalmol 2010; 94: 1109.

16 Lillakas L, Tarita-Nistor L, Lam D, Brent M, Steinbacj M, Gonzalez E. The effect of type-font on reading performance in age-related macular degeneration. ARVO 2011 (abstract 264). 